\title{
PAH IN VECTORIZED THREE DIMENSIONAL MONTE CARLO DUST RADIATIVE TRANSFER MODELS
}

\author{
R. Siebenmorgen ${ }^{1}$, F. Heymann ${ }^{2}$ and E. Krügel ${ }^{3}$
}

\begin{abstract}
We present a Monte Carlo (MC) radiative transfer code for complex three dimensional dust distributions and include transiently heated PAH. The correctness of the code is confirmed by comparison with benchmark results. The method makes use of the parallelization capabilities of modern vectorized computing units like graphic cards. The computational speed grows linearly with the number of graphical processing units (GPU). On a conventional desktop PC, our code is up to a factor 100 faster when compared to other MC algorithms. As an example, we compute the dust emission of proto-planetary disks. We simulate how a mid-IR instrument mounted at a future $42 \mathrm{~m} \mathrm{ELT}$ will detect such disks. Two cases are distinguished: a homogeneous disk and a disk with an outward migrating planet, producing a gap and a spiral density wave. We find that the resulting mid-IR spectra of both disks are almost identical. However, they can be distinguished at those wavelengths by coronographic, dual-band imaging. Finally, the emission of PAHs exposed to different radiation fields is computed. We demonstrate that PAH emission depends not only on the strength but also strongly on the hardness of the radiation, a fact which has often been neglected in previous models. We find that hard photons $(>20 \mathrm{eV}$ ) easily dissociate all PAHs in the disks of T Tauri stars. To explain the low, but not negligible detection rate $(<10 \%)$ of PAHs in $\mathrm{T}$ Tau disks, we suggest that turbulent motions act as a possible path for PAH survival.
\end{abstract}

\section{Dust model}

In the dust model we consider: large $(60 \AA<\mathrm{a}<0.2-0.3 \mu \mathrm{m})$ silicate (Draine 2003) and amorphous carbon (Zubko et al. 2004) grains and small graphite

\footnotetext{
${ }^{1}$ ESO, Karl-Schwarzschild-Str. 2, 85748 Garching b. München, Germany

${ }^{2}$ Astronomisches Institut, Ruhr-Universität Bochum, Universitätsstra. 150, 44801 Bochum, Germany and ESO

3 MPI für Radioastronomie, Auf dem Hügel 69, Postfach 2024, 53010 Bonn, Germany
} 
$(5<a<80 \AA)$ grains. For both, we apply a power law size distribution: $n(a) \propto$ $a^{-3.5}$ and absorption and scattering cross-sections are computed with Mie theory. In addition we include PAHs with 30 and $200 \mathrm{C}$ atoms. For the absorption cross section (Draine 2010; Malloci 2010; Verstraete 2010) we follow at photon energies between 1.7-15 eV Malloci et al. (2007, their Fig. 4). At low frequency, we take a cross section cut-off given as an average of neutral (Schutte et al. 1993) and ionized (Salama et al. 1996) species. For hard photons, beyond the $17 \mathrm{eV}$ band, we scale the photo-absorption cross section of PAH up to the keV region to similar sized graphite particles. By computing cross sections above $100 \mathrm{eV}$, we consider an approximation of kinetic energy losses (Dwek \& Smith (1996) and apply it to all dust particles. With the advent of ISO and Spitzer more PAH emission features and more details of their band structures are detected (Tielens 2008). We consider 17 emission bands and take, for simplicity and as suggested by Boulanger et al. (1998) and Siebenmorgen et al. (1998), Lorentzian profiles. Parameters are listed in Siebenmorgen \& Krügel (2001) and calibrated using mid-IR spectra of starburst nuclei. For starburst galaxies, a SED library is computed (Siebenmorgen et al. 2007) and those models provide good fits to the SED of local galaxies and to PAH detected at high red shifts $(z \approx 3$, Efstathiou \& Siebenmorgen 2009). In the model, we use dust abundances of $[\mathrm{X}] /[\mathrm{H}](\mathrm{ppm})$ of: 31 for [Si], 150 [amorphous $\mathrm{C}], 50$ [graphite] and $30[\mathrm{PAH}]$, respectively; which is in agreement with cosmic abundance constrains (Asplund et al. 2009). We are in the process of upgrading the model to be consistent with the polarisation of the ISM (Voshchinnikov 2004).

\section{Monte Carlo method}

We compute the radiative transfer with a Monte Carlo (MC) technique, which allows to handle complicated geometries, by following the flight path of many random photons. The basic ideas of our method go back to Lucy (1999) and Bjorkmann \& Wood (2001). They are described and extended to the treatment of transiently heated PAHs in Krügel (2006). The space is partitioned into cubes and, where a finer grid is needed, the cubes are further divided into subcubes (cells). The star emits photon packages of constant energy $\varepsilon$, but different frequencies. A package entering on its flight path a cell may be absorbed there or scattered. The probability for such an interaction is given by the extinction optical depth along the path within the cell. When the package is scattered, it only changes direction determined in a probabilistic manner by the phase function. When it is absorbed, a new package of the same energy, but usually different frequency $\nu_{\text {new }}$ is emitted from the spot of absorption. The emission is isotropic. Each absorption event raises the energy of the cell by $\varepsilon$, and accordingly its temperature.

We were able to increase the computational speed of the code by up to two orders of magnitude by parallelizing it, i.e. by calculating the flight paths of a hundred or more photons simultaneously. This allows the treatment of complex geometries which were so far prohibited because of their excessive computer demands. To achieve parallelization, we had to slightly modify the original code. The latter calculates the new frequency $\nu_{\text {new }}$ of a package that is emitted 
after absorption using the cell temperature before absorption, but applies a correction for the new temperature due to Bjorkmann \& Wood (2001). We now omit this correction or in, mathematical terms, use Equation (11.67) instead of Equation (11.65) in Krügel (2006). As expected and borne out in tests, the correction may be neglected when the number of photons absorbed in a cell is not small. The vectorized MC code was developed during the $\mathrm{PhD}$ project of Heymann (2010). It was verified against benchmarks provided for stellar heated dust spheres by Ivezic et al. (1997), the ray tracing code by Krügel (2006) and axi-symmetrical disks by Pascucci et al. (2004). Generally, the computed SED agrees with the benchmarks to within a few percent. One example of such comparisons is shown in Figure 1 for a dust sphere with PAHs. The factor by which parallelization speeds up the computation scales almost linearly with the number of graphical processing units. We point out that particular attention had to be given to the choice of the random number generator where we chose the Mersene Twister algorithm (Matsumoto \& Nishimura 1998).

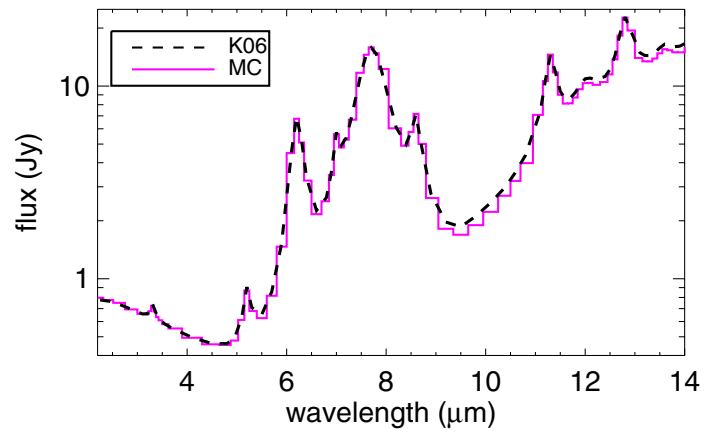

Fig. 1. Comparison of a SED of a stellar heated dust sphere of constant density and visual extinction to the star of $A_{\mathrm{V}}=10 \mathrm{mag}$ computed with a ray tracing method, as described in Krügel (2006), and the MC treatment of this work; both methods agree to within a few $\%$.

\section{Detection of proto-planetary disk structures}

Hydrodynamical simulations of proto-planetary disks with an orbiting planet show particular disk features (Masset et al. 2006). We present a three dimensional application of the MC code where a disk heated by a solar type star has a dust density which follows the one as given by Pascucci et al., and has in addition a gap between $3-8$ AU and a low density spiral structure. The optical depth along the midplane from 0.2 to $75 \mathrm{AU}$ is $10 \mathrm{mag}$. We simulate if such disk structures can be resolved at a distance of $50 \mathrm{pc}$ with a mid-IR instrument (Brandl et al. 2010) mounted at a future $42 \mathrm{~m}$ extreme large telescope (ELT); a project under study by ESO. The point spread function (PSF) of the ELT has resolution of 50 milliarcsec at $10 \mu \mathrm{m}$. In order to improve the contrast between star and disk, the instrument 

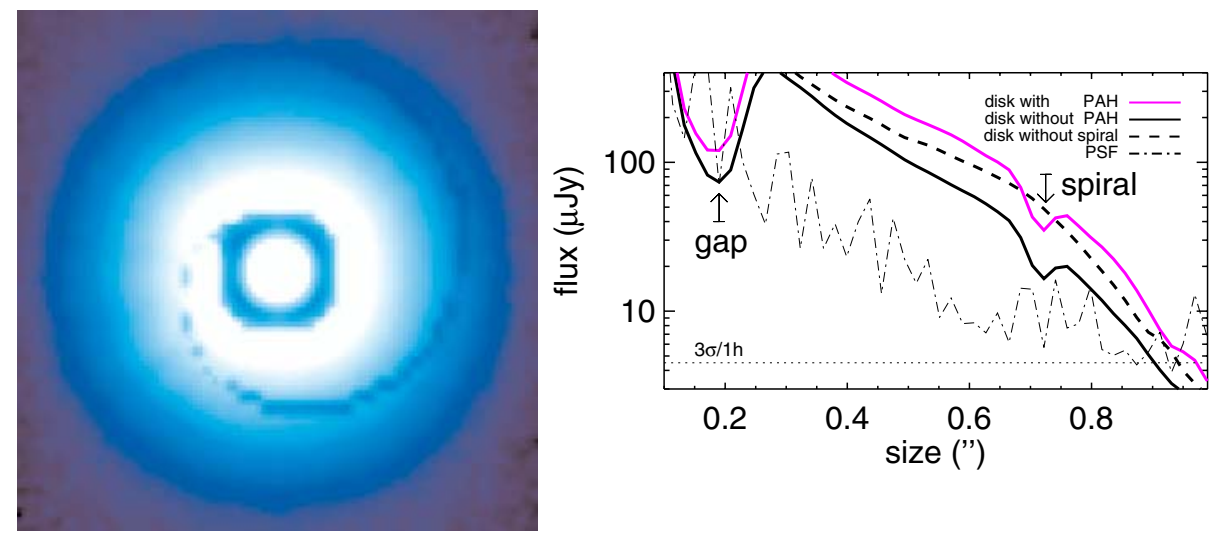

Fig. 2. Image at $11.3 \mu \mathrm{m}$ (left) together with flux profiles along the major axis (right). The $3 \sigma$ detection limit (dotted) and PSF (dash-dotted) is indicated. For the disk with (magenta) or without (black) PAH the gap and the spiral structure is well preserved. A homogeneous disk including PAHs is shown in dased for comparison.

will provide coronographic and dual band imaging modes. We choose band passes at 11.3 and $10 \mu \mathrm{m}$. The $11.3 \mu \mathrm{m}$ emission is shown in Figure 2 together with the flux profile along the major axis. Profiles of such a disk with and without PAHs and that of a homogeneous disk are computed. The $3 \sigma$ detection limit after $1 \mathrm{~h}$ integration is given assuming background limited performance of the instrument. The models predict that the detailed structure of such a proto-planetary disk can be well detected.

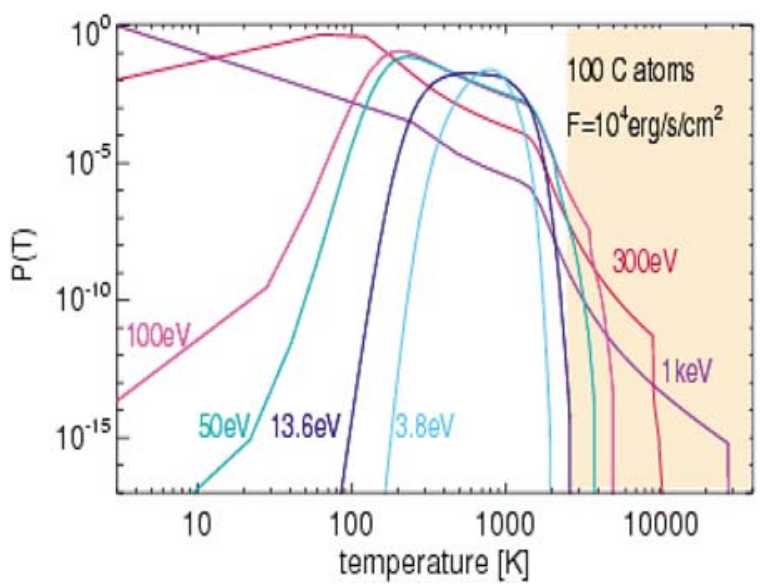

Fig. 3. The temperature distribution $P(T)$ of a PAH with $100 \mathrm{C}$ atoms exposed to monochromatic radiation with $h \nu=3.8,13.6,50,100,300 \mathrm{eV}$ and $1 \mathrm{keV}$ in a constant heating bath of flux $F=10^{4} \mathrm{erg} \mathrm{s}^{-1} \mathrm{~cm}^{-2}$ (Siebenmorgen \& Krügel 2010). The shaded area marks temperatures above the sublimation temperature of graphite. 


\section{Destruction and survival of PAH in T Tauri disks}

Despite the fact that from the stellar heating one would expect to detect PAH in the disks of $\mathrm{T}$ Tauri, one find them rather seldom, in less than $10 \%$ of $\mathrm{T}$ Tauri (Geers et al. 2006). In order to explain this fact we present a simplified scheme to estimate the location from the primary heating source at which the PAH molecules become photo-stable (Siebenmorgen \& Krügel 2010). T Tauri stars have beside photospheric emission also a far ultraviolet (FUV), an extreme ultraviolet (EUV) and an X-ray component with a fractional luminosity of about $1 \%, 0.1 \%$ and $0.025 \%$, respectively. Such hard photons are very efficient in dissociating PAHs. The temperature distribution, $P(T)$, of PAHs after hard photon absorption is shown in Figure 3. It demonstrates that $P(T)$ depends strongly on the hardness and spectral shape of the exciting radiation field, a fact which is often neglected in computations of the PAH emission. After photon absorption, a highly vibrationally excited PAH may relax through emission of IR photons or, if sufficiently excited, lose atoms (Omont 1986 \& Tielens 2005) for a textbook description). We find that hard photons (EUV and X-ray) would destroy all PAHs in the disk of $\mathrm{T}$ Tauri stars; whereas soft photons with energies $<20 \mathrm{eV}$ dissociate PAHs only up to short distances from the star (1-2 AU). As a possible path for PAH-survival turbulent vertical motions are suggested. They can replenish or remove PAHs from the reach of hard photons. In our treatment the presence of gas is considered which is ionized at the top of the disk and neutral at lower levels. A view of the scheme is shown as vertical cut along the midplane in Figure 4.

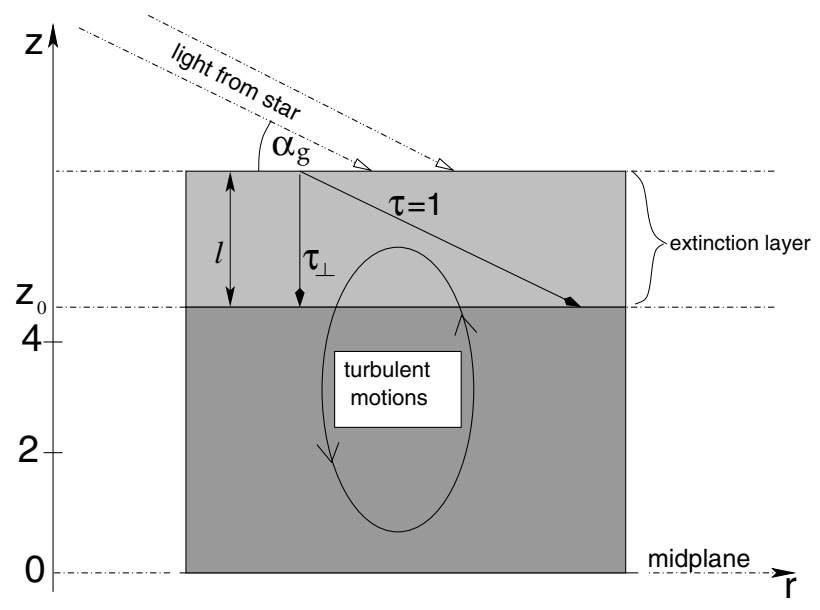

Fig. 4. Of each radiation components (photosphere, FUV, EUV and X-rays) of the fiducial T Tauri star about $90 \%$ is absorbed in what we call extinction layer. The optical depth from its bottom to the star is one and in the vertical direction equal to the grazing angle $\alpha_{\mathrm{g}}$. The height of its lower boundary $z_{0}$ declines with the radius, but its geometrical thickness is rather constant $(\ell \approx 0.5 \mathrm{H}$, for details see Siebenmorgen \& Krügel 2010). Vertical motions may either remove PAH from the extinction layer quickly enough for survival or replenish PAH from below. 


\section{References}

Asplund, M., Grevesse, N., Sauval, A.J., \& Scott, P., 2009, ARA\&A, 47, 481

Bjorkman, J.E., \& Wood, K., 2001, ApJ, 554, 615

Boulanger, F., Boisssel, P., Cesarsky, D., \& Ryter, C., 1998, A\&A, 339, 194

Brandl B., et al., 2010, SPIE, 7735-86, in press

Draine, B.T., 2003, ApJ, 598, 1026

Draine, B.T., 2011, this volume

Dwek, E., \& Smith, R.K., 1996, ApJ, 459, 686

Efstathiou, A., \& Siebenmorgen, R., 2009, A\&A, 502, 541

Geers, V.C., Augereau, J.-C., Pontoppidan, K.M., et al., 2006, A\&A, 459, 545

Heymann, F., 2010, Ph.D. Thesis, University of Bochum

Ivezic, Z., Groenewegen, M.A.T., Menshchikov, A., et al., 1997, MNRAS, 291, 121

Krügel, E., 2006, An introduction to the Physics of Interstellar Dust (IoP)

Lucy, L.B., 1999, A\&A, 344, 282

Malloci, G., Joblin, C., \& Mulas, G., 2007, A\&A, 462, 627

Malloci, G., 2011, this volume

Masset, F.S., Morbidelli, A., Crida, A., \& Ferreira, J., 2006, ApJ, 642, 478

Matsumoto, M., \& Nishimura, T., 1998, ACM Trans. Model. Comp. Simul., 8, 330

Micelotta, E.R., Jones, A.P., \& Tielens, A.G.G.M., 2010, A\&A, 510, A36

Omont, A., 1986, A\&A, 166, 159

Pascucci, I., Wolf, S., Steinacker, J., et al., 2004, A\&A, 417, 793

Salama, F., Bakes, E.L.O., Allamandola, L.J., \& Tielens, A.G.G.M., 1996, ApJ, 458, 621

Schutte, W.A., Tielens, A.G.G.M., \& Allamandola, L.J., 1993, ApJ, 415, 397

Siebenmorgen, R., Natta, A., Krügel, E., \& Prusti, T., 1998, A\&A, 339, 134

Siebenmorgen, R., Krügel, E., \& Laureijs, L., 2001, A\&A, 377, 735

Siebenmorgen, R., \& Krügel, E., 2007, A\&A, 461, 445

Siebenmorgen, R., \& Krügel, E., 2010, A\&A, 511, A6

Tielens, A.G.G.M., 2005, The Physics and Chemistry of the Interstellar Medium

(Cambridge Univ. Press)

Tielens, A.G.G.M., 2008, ARA\&A, 46, 289

Verstraete, L., 2011, this volume

Voshchinnikov, N.V., 2004, Optics of cosmic dust I, ASPR, 12, 1

Zubko, V., Dwek, E., \& Arendt, R.G., 2004, ApJS, 152, 211 